coplasia. O tratamento para este tipo de lesão passa por a cessação tabágica e a excisão cirúrgica, o eletrocautério, a criocirurgia ou a ablação por laser CO2. Perante a extensão, a localização da lesão e as características histológicas da lesão optou-se por ablação por laser CO2, com uma redução franca do tamanho da lesão. Este tipo de laser cujo mecanismo de ação é através do calor, constitui uma boa opção terapêutica uma vez que provoca uma ablação dos tecidos por desidratação, e hemorragia ligeira sem lesar os tecidos adjacentes, pelo que pode ser utilizada em lesões de grandes dimensões sem necessidade de enxertos O pós-operatório decorreu sem intercorrências e atualmente o doente encontra-se muito satisfeito, com vigilância apertada a cada 6 meses, dada elevada probabilidade de recorrências e a possibilidade de transformação maligna

http://doi.org/10.24873/j.rpemd.2018.11.277

\section{\#040 Impacto do Eixo II no Eixo I: a propósito de um caso clínico}

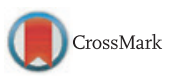

Paula Moleirinho Alves*, Pedro Cebola, Ângela Leal, André Mariz Almeida, Eduardo Januzzi

Pratica Clinica Privada; Instituto Universitário Egas Moniz (IUEM); Centro de Investigação Interdisciplinar Egas Moniz (CiiEM); Escola Superior de Saúde Egas Moniz (ESSEM)

Introdução: Um dos mais usados sistemas de classificação o Research Diagnostic Criteria for temporomandibular disorders baseia-se num modelo biocomportamental da dor com dois eixos: sinais e sintomas físicos (eixo I) e fatores psicológicos (eixo II). Os fatores psicológicos como a catastrofização da dor, o sofrimento psíquico, as crenças relacionadas à perceção dolorosa, humor deprimido ou ansioso e coping passivo estão relacionados com o aumento da perceção da dor, aumento dos níveis de incapacidade e distúrbios do movimento em pacientes com disfunção temporomandibular dolorosa crónica. Consideramos que a consciência somática é um fator sensório-discriminativo importante a ter em consideração neste grupo de pacientes.

Descrição do caso clínico: Paciente de 23 anos, género feminino com histórico de cefaleia generalizada desde há 2 anos. Apresentava cefaleia, dor miofascial (III) generalizada na região da cabeça e cervical, bruxismo e fatores psicológicos importante relacionados catastrofização da dor e ansiedade. No período correspondente ao início da manifestação dos sintomas dolorosos sofreu alterações psicossociais e familiares relevantes. O plano de tratamento consistiu em terapia cognitivo-comportamental, medicação, goteira oclusal de estabilização, fisioterapia e encaminhamento para psicoterapia.

Discussões e conclusões: Dois meses após o início da aplicação do plano de tratamento a paciente consegue identificar as situações psicológicas desencadeantes de exacerbação de sintomas, apresenta ausência de cefaleias e redução significativa da dor miofascial (II) que se encontra localizada no masséter e temporal, e é um claro exemplo do funcionamento do modelo biopsicossocial.

http://doi.org/10.24873/j.rpemd.2018.11.278
\#041 A relevância da abordagem multidisciplinar nas Disfunções Temporomandibulares

Pedro Cebola*, Paula Moleirinho Alves, Shanna Soares, André Mariz Almeida, Eduardo Januzzi

Pratica Clinica Privada; Instituto Universitário Egas Moniz (IUEM); Centro de Investigação Interdisciplinar Egas Moniz (CiiEM); Escola Superior de Saúde Egas Moniz (ESSEM)

Introdução: As disfunções temporomandibulares revelam uma elevada complexidade com uma etiologia multifactorial e afetam uma quantidade relativamente elevada da população mundial requerendo uma avaliação e diagnóstico multidisciplinar por parte da equipa clínica. O médico dentista enquanto profissional de primeira linha é responsável por realizar o diagnóstico, identificar factores de risco e realizar técnicas de abordagem multidisciplinar reversíveis e pouco invasivas. $\mathrm{O}$ Fisioterapeuta tem como objetivos reduzir a dor músculo-esquelética, promover o relaxamento muscular, reduzir a hiperatividade muscular, restaurar a função e controlo motor e reforçar a terapia cognitivo comportamental.

Descrição do caso cliníco: Paciente de 42 anos, sexo feminino, refere disfunção temporomandibular. Diagnóstico: hipomobilidade articular (29 mm), bruxismo de vigília e do sono, deslocamento de disco sem redução com limitação da abertura, crepitação, artralgia, dor miofascial no masséter com dor referida para outras regiões anatómicas. O Plano de tratamento consistiu em terapia cognitivo comportamental, farmacoterapia, goteira oclusal de estabilização, infiltração com Osteonil ${ }^{\circledR}$ na articulação temporomandibular, aplicação de técnicas de mobilização articular, técnicas neuromusculares e miofasciais Discussões e Conclusões: Após 2 meses do inicio da plano de tratamento a paciente apresenta já uma abertura de $38 \mathrm{~mm}$, diminuição da intensidade da crepitação, a artralgia mantem-se mas apenas em abertura máxima, dor miofascial apenas quando estimulada. Mantém-se no entanto o deslocamento do disco sem redução, mas constatou-se uma melhoria significativa da qualidade de vida da paciente.

http://doi.org/10.24873/j.rpemd.2018.11.279

\#042 Sobredosagem acidental de Metotrexato em doente com Artrite Reumatóide

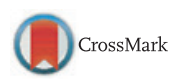

André Pereira*, Ana Teresa Tavares, Natacha Ribeiro, Luís Sanches Fonseca, Rosário Marques, Marcelo Prates

Hospital de São José

Introdução: O metotrexato está indicado no tratamento da artrite reumatóide em casos graves ou re-fratários a outros tratamentos e a posologia varia entre 7,5 a 20mg semanalmente, per os. Estão descritos casos de toxicidade potencialmente fatais relacionados com a toma in-correcta deste fármaco, nomeadamente toma diária. A toxicidade inclui reações cutâneas graves, estomatite ulcerosa, osteonecrose, supressão medular ou toxicidade hepática. O interesse deste caso clínico prende-se com o facto de ser uma doente com artrite reumatóide em 
agravamento, que sob metotrexato desenvolveu um quadro de mucosite ulcerosa grave.

Descrição do caso clínico: Mulher de 62 anos com Artrite Reumatóide e Diabetes mellitus, medicada com Predniso-lona, Leflunamida, Metformina e Glibenclamida e sem alergias medicamentosas conheci-das. Observada em Consulta de Doenças Autoimunes por agravamento de ciatalgia direita no contexto de Artrite Reumatóide. Foram prescritos 12,5 mg de Metotrexato semanal per os que, por erro, tomou diariamente. Após um mês recorre à urgência por ulcerações orais e da orofaringe dolorosas, com uma semana de evolução, dificultando a deglutição e ali-mentação. Analiticamente apresentava pancitopénia, aumento de enzimas hepáticas e da Proteína C Reativa. Objectivamente apresentava edema e ulcerações friáveis e dolorosas em toda a mucosa jugal e lábios. Não evidenciava lesões cutâneas. Na presunção de re-ação adversa a sobredosagem de metotrexato, a doente foi internada no Serviço de Me-dicina Interna com suspensão de toda a medicação, iniciando Ácido Fólico, manipulado de lidocaína/bicarbonato/ nistatina em suspensão oral e piperacilina/tazobactam por neu-tropénia febril. Foi transfundida com uma unidade de concentrado eritrocitário e dois pools plaquetários e fez dieta

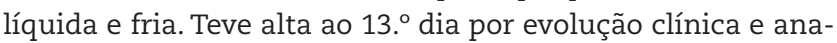
lítica favoráveis. Atualmente está sem queixas articulares ou orais sob Leflunamida e Prednisolona.

Discussão e conclusões: Os efeitos tóxicos do metotrexato podem relacionar-se em frequência e gravidade com a posologia, pelo que estes doentes devem ser mantidos sob vigilância clínica e laboratori-al. A maioria das reações adversas é reversível se existir uma detecção precoce, redução ou suspensão terapêutica. É fundamental informar os doentes sobre os potenciais bene-fícios e riscos deste fármaco, bem como alertá-los para a importância da adesão à poso-logia prescrita. http://doi.org/10.24873/j.rpemd.2018.11.280

\#043 Implantoplastia como opção de tratamento da peri-implantite: série de casos clínicos

Orlando Martins*, Sérgio Matos, João Carlos Ramos, Francisco Caramelo, Isabel Poiares Baptsta

Área de Medicina Dentária, Faculdade de Medicina, Universidade de Coimbra; Laboratório de Bioestatística e Informática Médica, Faculdade de Medicina, Universidade de Coimbra

Introdução: O tratamento da peri-implantite não é previsível nem consensual. Os resultados a longo termo da abordagem regenerativa têm uma evidência limitada e são mais onerosos que a ressetiva. A implantoplastia consiste na remoção da camada mais externa do implante contaminado e subsequente polimento.

Descrição do caso clínico: Nesta análise retrospetiva de uma série de casos foram incluídos 8 pacientes diagnosticados com peri-implantite (17 implantes) e tratados através de implantoplastia. Peri-implantite foi definida como profundidade de sondagem $\geq 5 \mathrm{~mm}$, perda óssea marginal $\geq 2 \mathrm{~mm}$, hemorragia à sondagem e/ou supuração. Critérios de inclusão: a) implante imóvel; b) sem sobrecarga oclusal; c) índice de placa bacte- riana $<1$; d)não fumador ou $\leq 10$ cigarros/dia; e) sem patologia sistémica que influencie resultado da terapia. Para cada variável analisada foi determinada a média e desvio padrão na baseline e 12 meses. Através do teste de Wilcoxon avaliaram-se diferenças entre 0 e 12 meses $\left(\right.$ IBM $^{\circledR}$ SPSS $^{\circledR} \mathrm{V} 24$, nível de significância de 0.05). Na baseline as variáveis analisadas foram: profundidade de sondagem $=5.15 \pm 0.48 \mathrm{~mm}$; hemorragia à sondagem $=0.89 \pm 0.15$; supuração $=0.05 \pm 0.07$; recessão gengival $=0.47 \pm 0.6 \mathrm{~mm}$; nível clínico de inserção $=5.61 \pm 0.46 \mathrm{~mm}$. Cirurgicamente realizou-se um retalho de espessura total, removeu-se o tecido de granulação com curetas e procedeu-se à implantoplastia com brocas diamantadas esféricas (turbina) e broca de Arkansas (contra-ângulo). O retalho foi reposicionado apicalmente e suturado. Aos 15 dias removeu-se a sutura. Pacientes controlados nos meses 1, 3, 6, 9 e 12. Aos 12 meses verificou-se uma redução estatisticamente significativa da profundidade de sondagem $(2.80 \pm 0.36 \mathrm{~mm})(\mathrm{p}=0.012)$; hemorragia à sondagem $(0.08 \pm 0.1)(p=0.011)$; recessão gengival $(1.45 \pm 0.89 \mathrm{~mm})(\mathrm{p}=0.012)$ e nível clínico de inserção $(4.25 \pm 0.85 \mathrm{~mm})(\mathrm{p}=0.011)$.

Discussão e conclusões: Nesta série de casos obteve-se a resolução da patologia, com uma taxa de sobrevivência de 100\%, aos 12 meses. A melhoria dos parâmeros clínicos e a taxa de sobrevivência estão de acordo com os resultados de Romeo et al em 2005. Outros estudos apontam para taxas de sobrevivência de $87.2 \%$, aos 108 meses. O aumento da recessão gengival tem como consequência a exposição da superfície do implante, o que pode ser problemático em áreas estéticas. Dentro das limitações desta análise retrospetiva concluiu-se que a implantoplastia permitiu a resolução da patologia. http://doi.org/10.24873/j.rpemd.2018.11.281

\#044 Prótese Esquelética por Sinterização Directa de Metal por Laser - Caso Clínico

Isabel Magalhães Gomes*, João Paulo Martins, Luis Pires Lopes

FMDUL

Descrição do caso clinico: Uma paciente do sexo feminino com 50 anos de idade e sem antecedentes relevantes compareceu na consulta de Reabilitação Oral da FMDUL para reabilitação dos espaços edêntulos. Após exame clínico e radiográfico confirmou-se não serem necessários tratamentos pré-protéticos, estando a paciente em condições de proceder ao tratamento reabilitador das suas desdentações, classe III modificação 1 de Kennedy-Applegate, na maxila e na mandíbula. Realizaram-se as impressões preliminares e procedeu-se à análise dos modelos de estudo. Após terem sido executadas as preparações pré-protéticas nos dentes pilares foram realizadas as impressões definitivas. Os modelos de trabalho em gesso foram digitalizados. Com o software 3Shape foi desenhada a estrutura do esqueleto metálico, sendo esta informação enviada via internet para o centro de produção (Phibo). Neste, a estrutura metálica em Cr-Co foi confeccionada pela técnica de sinterização directa de metal por laser (DMLS). Após prova da estrutura decorreu a sua acrilização e acabamento. 